\title{
Pseudopotential in resonant regimes
}

\author{
Ludovic Pricoupenko \\ Laboratoire de Physique Théorique de la Matière Condensée, \\ Université Pierre et Marie Curie, 4 place Jussieu, 75252 Paris Cedex 05, France.
}

(Dated: September 10, 2018)

\begin{abstract}
The zero-range potential approach is extended for the description of situations where two-body scattering is resonant in arbitrary partial waves. The formalism generalizes the Fermi pseudopotential which can be used only for $s$-wave broad resonances. In a given channel, the interaction is described either in terms of a contact condition on the wave function or with a family of pseudopotentials. We show that it is necessary to introduce a regularized scalar product for wave functions obtained in the zero-range potential formalism (except for the Fermi pseudopotential). This metrics shows that the geometry of these Hilbert spaces depends crucially on the interaction.
\end{abstract}

PACS numbers: 03.65.Nk,03.75.Ss,05.30.Fk,34.50.-s

\section{INTRODUCTION}

\section{A. Zero-range approach in ultracold physics}

The roots of the zero-range potential approach lie in the works on the neutron-proton interaction in nuclear physics. First, Bethe and Peierls [1] showed that it was possible to get numerous scattering properties on deuteron by replacing the neutron-proton force with a suitable boundary condition on the wave function in the $s$-wave channel. One year after, Fermi 2] introduced the idea of a zero-range effective potential allowing a Born approximation in the computation of the scattering cross section between a slow neutron and a proton. Equivalence between these two approaches was developed further by Breit [3] and led to the final formulation of the so-called Fermi pseudopotential 4]. The fact that the range of the nuclear force $\left(\simeq 210^{-15} \mathrm{~m}\right)$ is much less than the $s$-wave neutron-proton triplet scattering length $\left(\simeq 4.310^{-15} \mathrm{~m}\right)$ was a key ingredient for the success of this approach.

More than half a century after these pioneer works, zero-range pseudopotentials appear as reference tools in the physics of ultracold atoms. There are several reasons for this renewed interest. First, the mean interparticle distance in ultracold gases is very large as compared to the range of interatomic forces (denoted by $R$ ), so that two-body collisions occur at energies very much smaller than the characteristic high-energy scale $\hbar^{2} / m R^{2}(m$ is the particle mass). Consequently, the full two-body potential contains useless informations (deep bound states, hard-core repulsion ...) for the study of the ultracold dilute gases. In this respect, the pseudopotentials which by construction reproduce the correct scattering phase shift at small energy, contain the minimal number of parameters for modeling two-body collisions. A second key property is that for processes of vanishly small energies, a large class of pseudopotentials give the correct results at the first order Born approximation while this approximate treatment is not possible using the "real" two-body potential. Hence, mean-field schemes like the Gross-Pitaevskii and the Bogoliubov approaches [5, 6] for the Bose gas or BCS 7] for the dilute two-component Fermi gas can be implemented using the Fermi pseudopotential. This idea has been generalized by introducing a family of pseudopotentials equivalent to the Fermi pseudopotential: the $\lambda$-potentials [8]. Varying the free parameter $\lambda$ does not change the exact treatment of the two-body problem, while the Born approximation can be adjusted to the exact solution for a finite collisional energy. In the case of the ultracold Bose gas and using the Hartree-Fock-Bogoliubov formalism, the $\lambda$-potential permits to self-consistently take into account the back action of the excitations on the condensate in three-dimensional systems 8 and also in quasi-two-dimensional (2D) traps [9]. Scattering properties in low dimensional configurations are another powerful application of the zero-range potential approach. For example, in the case of $s$-wave scattering, the Bethe-Peierls boundary condition on the $3 \mathrm{D}$ wave function is a very efficient way for finding the scattering amplitude of two particles trapped in planar or linear atomic waveguides 10, 11, 12. The important concept of tunable interaction in quasi-one-dimensional and quasi-two-dimensional systems has been discovered thanks to this approach [10, 11]. The few-body problem is another area of research where the zero-range potential approach has already proven to be very fruitful. Exact results have been found for three and four particles interacting by pairs in the $s$-wave channel $13,14,15,16]$. These studies are of first importance in the context of the resonant $s$-wave scattering regime obtained with Feshbach resonances [17]. In these experiments the scattering length can be tuned at will, so that it is possible to achieve the BCS-BEC crossover of the two-component Fermi gas 18, 19, 20, 21, 22]. The zero-range approach permits us to evaluate the lifetime (which is remarkably large in the neighborhood of the resonance) for the weakly bound molecules that appear in the BEC phase and also to find the value of the molecule-molecule scattering length in terms of the two-body scattering length 14. For the unitary quantum gas (infinite two-body scattering length) exact results have been found for the few- and many-body problems [23, 24]. These studies show clearly that the zero-range potential approach is an 
efficient tool beyond the two-body physics and permits us to obtain non trivial properties in the few- and manybody problems.

More generally, study of strongly correlated systems obtained in the vicinity of scattering resonances is actually one of the most challenging directions in the field of ultracold atoms. These systems are not only interesting in themselves but also permit accurate studies of issues raised in the quantum many-body problem. The BCSBEC crossover in two-component Fermi gases is an example of such a situation [25, 26, 27]. The domain now enlarges to resonant scattering in $p$-wave [28, 29, 30, 31, 32] and $d$-wave channels 33] allowing possible studies of the BCS-BEC crossover in channels of high angular momentum in the near future. Major interest in these systems stems from the fact that the strength of correlations can be tuned arbitrarily while the mean interparticle distance remains large with respect to the range of interatomic forces $R$ (for example, the $s$-wave scattering length can be adjusted to several orders of magnitude larger than $R$ ). Consequently, one can expect that short range physics is not directly involved in a large class of many-body properties which can then be described only in terms of the low energy two-body behavior. It is worth pointing out that this fundamental feature has been verified in the BCS-BEC crossover of the two-component Fermi gas. For this reason, the zero-range approach which is parametrized only by the two-body low energy physics is a very appealing tool for studying these regimes. Actually, the state of the art is as follows: broad $s$-wave resonances (like the one in ${ }^{6} \mathrm{Li}$ at $835 \mathrm{G}[20]$ ) can be accurately parametrized using the Fermi pseudopotential, and narrow $s$-wave resonance [34] can be studied using a generalization of the Bethe-Peierls boundary condition 15. Recently, a general zero-range potential approach has been introduced for $p$-wave resonances [35] and the aim of this paper is to extend this formalism for a description of resonant scattering regime in arbitrary partial waves.

\section{B. Which generalization of actual pseudopotentials is needed?}

The idea of replacing a true finite range two-body potential by a zero-range pseudopotential acting on each partial wave has been developed by Huang and Yang [36] in the context of the hard-sphere model. However, due to the importance of the short range behavior in this system, pseudopotentials describing three-, four- and many-body correlations were also proposed for modeling the system beyond the dilute limit. The actual situation in ultracold atoms is radically different as the mean interparticle distance is very much larger than the range of interatomic forces, and two-body pseudopotentials are sufficient to describe the low-energy behavior for the many-body system even in strongly correlated regimes. Moreover as the hard-sphere model cannot support resonances, the tools developed in Ref. [36] cannot be used for our purpose. Recently, a generalization of the Fermi pseudopotential for arbitrary partial waves and scattering phase shifts has been proposed using delta-shell potentials in the limit of small shell radius [37], by the way correcting a mistake of Ref. [36]. Boundary conditions associated with the zero-range approach in Ref. [37] are defined for scattering states in free space and depend explicitly on their wave number $k$. Consequently, this approach applies only in time-independent situations. Instead in the present paper, we show that in resonant regimes, general (that is, energy independent) boundary conditions can be found. Another feature of the pseudopotentials in Ref. [37] is that their action is defined on the radial wave function of the specified partial wave. However, it is of interest when dealing with wave functions of arbitrary symmetry (for example in the presence of an anisotropic external potential) to have the full expression of the pseudopotential, that is, an expression which contains implicitly the projection operator over the interacting channel (denoted in the following by $\Pi_{l}$ for the channel of angular quantum number $l$ ).

\section{Organization of the paper}

The paper is organized as follows: in the first part, the regime of interest (two-body resonant scattering) is introduced and characterized in each partial wave channel by a set of two parameters in the scattering phase shift. In the second part, the different tools of the zero-range approach in this regime are derived. We show that the problem can be defined in terms of boundary conditions on the wave function which generalize the method introduced by Bethe and Peierls. We express these boundary conditions in spherical coordinates and also in Cartesian coordinates using the symmetric trace free tensors used in the usual multipolar expansion. This way of defining the zero-range approach permits us to include quite easily within the expression of the pseudopotential both the projection operator $\Pi_{l}$ and the $l$ th derivatives of the delta distribution. We show that in each interacting channel there exists a family of pseudopotentials generated by a free parameter, thus generalizing the $\lambda$-potential approach 8, 35. The third and last part, illustrates the formalism. We find the expression of the two-body scattering states from the pseudopotential and the Green's function method. By choosing a specific value of the parameter $\lambda$, this exact result is also obtained in the first order Born approximation for two-body processes at finite energy. The pseudopotential of Ref. 37] is recovered as a particular limit of the present approach. Finally, the scattering states and also the low-energy bound states are found to be not mutually orthogonal in the zero-range scheme (except for the Fermi pseudopotential). We introduce then a regularized scalar product which solves this inconsistency. We consider the low-energy bound states supported by the pseudopotential and show that the new 
scalar product leads to normalization constants which coincide with the results given by the method based on the analyticy of the scattering amplitude [38].

\section{ZERO-RANGE FORMALISM IN THE RESONANT REGIME}

\section{A. Regime of interest}

Let us consider two particles in the absence of an external potential interacting via a short range isotropic potential $U(\vec{r})(\vec{r}$ being the relative coordinates between the two particles). We denote their reduced mass by $\mu$. In order to simplify the discussion and without loss of generality, we work in the center-of-mass frame. The asymptotic form of the scattering wave function $\Psi_{\vec{k}}(\vec{r})$ with relative wave vector $\vec{k}$ and collisional energy $E=\frac{\hbar^{2} k^{2}}{2 \mu}$ defines the partial amplitudes $f_{l}$ in each partial wave channel of angular quantum number $l$ through the relation:

$$
\Psi_{\vec{k}}(\vec{r})=\exp (i \vec{k} \cdot \vec{r})+\sum_{l=0}^{\infty}(2 l+1) \mathcal{P}_{l}\left(\vec{n} \cdot \vec{n}_{k}\right) f_{l} \frac{\exp (i k r)}{r}
$$

where $k r \gg 1, \vec{n}=\vec{r} / r, \vec{n}_{k}=\vec{k} / k$ and $\mathcal{P}_{l}$ is the Legendre polynomial of degree $l$. The partial amplitudes can be also expressed in terms of the phase shift $\delta_{l}$ with:

$$
f_{l}=\frac{1}{2 i k}\left(\exp \left(2 i \delta_{l}\right)-1\right) .
$$

Typical situations in ultracold atoms corresponds to binary collisions of particles having small enough relative velocities to ensure $k R \ll 1$, where $R$ is the potential range. For two neutral alkali atoms in their ground state, the asymptotic form of $U(\vec{r})$ is a van der Waals tail: $U(\vec{r}) \simeq C_{6} / r^{6}$ and the criterion for low-energy scattering processes (collisional energy $E \ll E_{R}$ ) is fixed by [39, 40]:

$$
R=\left(\frac{2 \mu C_{6}}{\hbar^{2}}\right)^{1 / 4} \quad ; \quad E_{R}=\frac{\hbar^{2}}{2 \mu R^{2}}
$$

Standard values for $R$ (and $E_{R}$ ) range from $\sim 3.3 \mathrm{~nm}$ for ${ }^{6} \mathrm{Li}\left(E_{R} \simeq 7.25 \mathrm{mK}\right)$ to $\sim 10 \mathrm{~nm}$ for ${ }^{133} \mathrm{Cs}\left(E_{R} \simeq\right.$ $32.2 \mu \mathrm{K})$. In this paper we consider situations where the phase shift can be parametrized in the following form:

$$
k^{2 l+1} \cot \delta_{l}(k)=-\frac{1}{w_{l}}-\alpha_{l} k^{2} .
$$

Eq.(44) corresponds to the so-called effective range approximation for a short range potential [41]: the parameter $w_{l}$ characterizes the Wigner threshold regime and $-2 \alpha_{l}$ is "the effective range" even if it has the dimension of a length only for $l=0$. For neutral particles interacting with a potential of asymptotic form $U(r) \simeq C_{n} / r^{n}$ $(r \rightarrow \infty)$ the threshold behavior is valid for $l<(n-3) / 2$ and the effective range approximation for $l<(n-5) / 2$. In ultracold atoms in their ground state the power law is given by $n=6$ so that the expansion in Eq.(4) is rigorous only in the $s$-wave channel ( $w_{0}$ is the scattering length), while in the $p$-wave channel only the first term in the expansion is justified. For $l \geq 2$, even the Wigner threshold regime given in Eq.(4) is not valid. However, we adopt this form as a generic way to parametrize the resonant regime in an arbitrary partial wave channel.

In broad $s$-wave resonances, the effective range is negligible $\left(\alpha_{0} \simeq 0\right)$ and $\left|w_{0}\right|$ is very large as compared to the potential range $R$. For $w_{0}>0, f_{0}$ has a pole associated to a shallow bound state of energy $-\hbar^{2} / 2 \mu w_{0}^{2}$. Its wave function coincides (for $r>R$ ) with the molecular state populated by pairs of particles in the BEC region of the BEC-BCS crossover. Remarkably, in the neighborhood of the resonance, the scattering cross section takes large values whatever the sign of the scattering length $w_{0}$. As explained in Ref. [38], in the channels $l>0$, this property is no longer verified, moreover the "effective range" parameter is essential for a modeling of the shape of the scattering amplitude and of the shallow bound state both. To define the resonant regime for $l>0$, we follow the discussion given in Ref. [38] and use the expression of the partial amplitude associated with the phase shift in Eq.(4):

$$
f_{l}(k)=-\frac{w_{l} k^{2 l}}{1+w_{l} \alpha_{l} k^{2}+i w_{l} k^{2 l+1}} .
$$

The resonant regime corresponds to situations where the parameter $\left|w_{l}\right|$ takes arbitrary large values, so that $\left|w_{l} \alpha_{l}\right| \gg R^{2}$. It can be shown [38] that describing the resonant regime by Eq. (15) is valid only for $\alpha_{l}>0$ (this assumption emerges also as a consequence of the regularized scalar product that we introduce in the last section). The main difference as compared to $s$-wave broad resonance is that the resonant character of the scattering cross section associated with Eq.(5) depends crucially on the sign of $w_{l}$. This property can be easily seen by writting Eq.(5) in the Breit-Wigner form:

$$
f_{l}=-\frac{1}{k} \frac{\Gamma / 2}{E-E_{r}+i \Gamma / 2},
$$

with $E_{r}=-\frac{\hbar^{2}}{2 \mu \alpha_{l} w_{l}}$ and $\Gamma=\frac{\hbar^{2} k^{2 l+1}}{\mu \alpha_{l}}$. For large and positive values of $w_{l}, E_{r}<0$ and the scattering amplitude has a pole which corresponds to the presence of a real bound state of vanishing energy $E_{B} \simeq E_{r}$. In this regime, $\left|f_{l}\right|^{2}$ does not present a resonant structure. However, for large and negative value of $w_{l}$, the bound state transforms into a long-lived quasi bound state which produces a resonance in the scattering cross section with $\left|f_{l}\right|^{2} \simeq 1 /\left|w_{l} \alpha_{l}\right|$ in the neighborhood of $E=E_{r}$. This feature is a consequence of the existence of the centrifugal barrier in the radial equation for $l>0$ (see Fig 1). It is worth pointing out that the resonant regime occurs for 


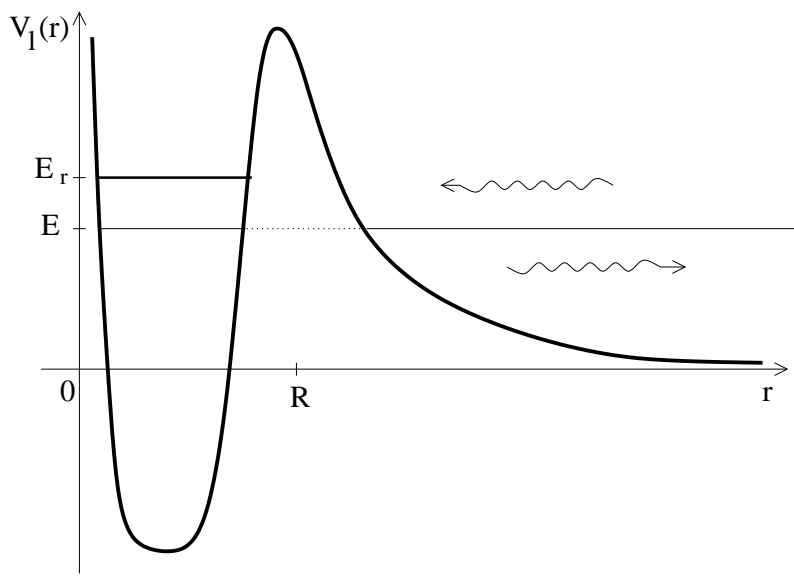

FIG. 1: Schematic representation of a two-body effective radial potential $V_{l}(r)=U(\vec{r})+\frac{\hbar^{2} l(l+1)}{2 \mu r^{2}}$ in a partial wave $l>0 . E_{r}$ is the energy of a quasi-bound state [4, 38] which leads to a resonant behavior in the two-body scattering for a collisional energy $E \simeq E_{r}$.

relative wave vectors $k$ verifying $k R \ll 1$, which justifies a zero-range potential approach.

In actual experiments on ultracold atoms, the resonant character in the two-body scattering is ensured using Feshbach resonances [42]. Interaction between atoms depends on the spin configuration of the two-body system and Feshbach resonances appear as a result of the coupling between a closed channel (supporting a shortrange molecular state) and an open channel (associated with the scattering states). The energy of the short-range two-body molecular state can be tuned by use of an external magnetic field. The scattering resonance occurs when the energy of the molecular state is in the vicinity of the collisional energy of the two scattering atoms. The molecular state in the closed channel depends on the short range properties of the system and is not described by the zero-range approach. Consequently, the bound state or quasi-bound state which appears in the zero-range model is associated only with the tail of the molecular state resulting from the coupling between the closed and open channels.

In the $s$-wave narrow Feshbach resonance (large and negative effective range) of ${ }^{23} \mathrm{Na}$ at $907 \mathrm{G} \mathrm{[34],} \alpha_{0} \simeq$ $26 \mathrm{~nm}$, and $R \simeq 4.5 \mathrm{~nm}$. In higher partial wave channels, scattering is not isotropic i.e. it depends on the angular quantum number $m$ of the state. For example the $p$-wave resonance in ${ }^{40} \mathrm{~K}[29](R \simeq 6.8 \mathrm{~nm})$ has been parametrized with the law (4) in the states $|m|=0,1$ as a function of the external magnetic field. There are two zero energy resonances $\left(E_{r}=0\right)$ very close to one each other at $198.84 \mathrm{G}$ (for $m=0)$ and 198.31 $\mathrm{G}$ (for $|m|=1$ ) with a width characterized respectively by $\alpha_{1} R \simeq 2.74$ and 2.81. Generalization of the zero range approach for taking into account this anisotropy adds technical details not essential in a first approach and in this paper, the discussion is centered on the modeling of isotropic interactions.

\section{B. Defining the zero-range approach}

In this section, the interacting potential $U(\vec{r})$ which can be neglected in the Schrödinger equation for $|\vec{r}|>R$ is replaced by a specific behavior of the wave function as the relative coordinates between the two interacting particles goes formally to zero. This behavior defines boundary conditions on the wave function which depend on the parametrization of the scattering phase shift in Eq. (4).

Outside the potential range $(r>R)$, the scattering wave functions $\left\{\Psi_{\vec{k}}(\vec{r})\right\}$ of the two-body problem are solutions of the free Schrödinger equation with the asymptotic behavior given by Eqs.(124):

$$
\Psi_{\vec{k}}(\vec{r})=\sum_{l=0}^{\infty} \mathcal{P}_{l}\left(\vec{n} \cdot \vec{n}_{k}\right) R_{k l}(r)
$$

where $R_{k l}(r)$ are the radial functions which can be expressed in terms of the two spherical Bessel functions as 43]:

$$
R_{k l}(r)=\mathcal{N}_{k l}\left(j_{l}(k r)-y_{l}(k r) \tan \delta_{l}(k)\right) .
$$

The zero-range approach amounts to considering the formal continuation of Eq. (8) for $0 \leq r \leq R$. As a consequence, using the behavior of $j_{l}(z)$ and $y_{l}(z)$ at the vicinity of the origin:

$$
\begin{aligned}
& j_{l}(z)=\frac{z^{l}}{(2 l+1) ! !}\left(1-\frac{z^{2} / 2}{1 !(2 l+3)}+\ldots\right) \\
& y_{l}(z)=-\frac{(2 l-1) ! !}{z^{l+1}}\left(1-\frac{z^{2} / 2}{1 !(1-2 l)}+\ldots\right)
\end{aligned}
$$

one can deduce the scattering states from the following boundary conditions of the wave function at $r=0$ :

$$
\lim _{r \rightarrow 0}\left(\frac{(2 l-1) ! !}{(2 l) ! !} \partial_{r}^{(2 l+1)}-\frac{k^{2 l+1}}{\tan \delta_{l}(k)}\right) r^{l+1} R_{k l}(r)=0 .
$$

This expression is a first step toward the formulation of the zero range approach, but does not have a general character in the sense that the quantum number $k$ appears explicitly. Instead, we search for a boundary condition which applies on any linear combination of scattering states with different collisional energies. This way, the formalism can be used when the collisional energy is not defined, as it can happen in time dependent situations. Using the parametrization of the phase shift in Eq.(4), one obtains from Eq.(11):

$$
\lim _{r \rightarrow 0}\left(\frac{(2 l-1) ! !}{(2 l) ! !} \partial_{r}^{(2 l+1)}+\alpha_{l} k^{2}+\frac{1}{w_{l}}\right) r^{l+1} R_{k l}(r)=0 .
$$


The idea is then to substitute the energy dependent term in Eq.(12) by the appropriate radial derivative. From Eqs.1012) we find the desired condition:

$$
\lim _{r \rightarrow 0}\left(\mathcal{D}_{l}+\frac{1}{w_{l}}\right) r^{l+1} R_{k l}=0
$$

where we have introduced the differential operator:

$$
\mathcal{D}_{l}=\frac{(2 l-1) ! !}{(2 l) ! !} \partial_{r}^{(2 l+1)}+(2 l-1) \alpha_{l} \partial_{r}^{2}
$$

We are now ready to define the zero-range approach in general situations. The wave function $\Psi$ of the interacting two-body system is expanded in each partial wave channel in terms of the spherical harmonics as:

$$
\left\langle\vec{r}\left|\Pi_{l}\right| \Psi\right\rangle=\sum_{m=-l}^{l} \frac{c_{\Psi}^{l m}(r)}{r^{l+1}} Y^{l m}(\Omega)
$$

where $\Pi_{l}$ is the projector on the subspace of angular quantum number $l$, and $(r, \Omega)$ are the spherical coordinates with $\Omega=(\theta, \phi)$. For $r \neq 0, \Psi(\vec{r})$ is a solution of the free Schrödinger equation (it implies that the multipolar radial functions $c_{\Psi}^{l m}(r)$ are regular functions of the interparticle distance $r$ ), and for an isotropic interaction, the potential term is replaced by the following boundary conditions on the wave function at $r=0$ :

$$
\lim _{r \rightarrow 0}\left(\mathcal{D}_{l}+\frac{1}{w_{l}}\right) c_{\Psi}^{l m}(r)=0 \quad-l \leq m \leq l .
$$

Eq.(16) generalizes the Bethe-Peierls approach [1] which applies in the $s$-wave channel when the effective range can be neglected $\left(\alpha_{0}=0\right)$ :

$$
\lim _{r \rightarrow 0} \frac{\partial_{r}(r \Psi)}{(r \Psi)}=-\frac{1}{w_{0}}
$$

where the limit $\left|w_{0}\right| \gg R$ is associated with a zero energy broad resonance.

Note that the generalization of Eqs. (16) to the case of an anisotropic two-body interaction is straightforward by introducing parameters that depend on the quantum number $m:\left\{w_{l m}, \alpha_{l m}\right\}$.

\section{Cartesian representation of the multipolar radial functions}

While the zero-range approach is now established in a general way by Eqs. 141516), it can be easier to use equivalent boundary conditions in the Cartesian coordinate system (for example when the system is inhomogeneous or anisotropic). Furthermore, as will be shown in the next section, using these conditions leads to a simple construction of the zero-range pseudopotential (in particular, derivatives of the delta distribution are easily introduced in Cartesian coordinates). In the following,

\begin{tabular}{|c|c|c|}
\hline \hline$l$ & $\mathcal{S}_{l}^{[\alpha \beta \ldots]}$ & $\mathcal{D}_{l}$ \\
\hline 0 & 1 & $\partial_{r}-\alpha_{0} \partial_{r}^{2}$ \\
\hline 1 & $n^{\alpha}$ & $\frac{1}{2} \partial_{r}^{3}+\alpha_{1} \partial_{r}^{2}$ \\
\hline 2 & $n^{\alpha} n^{\beta}-\frac{1}{3} \delta^{\alpha \beta}$ & $\frac{3}{8} \partial_{r}^{5}+3 \alpha_{2} \partial_{r}^{2}$ \\
\hline 3 & $n^{\alpha} n^{\beta} n^{\gamma}-\frac{1}{5}\left(n^{\alpha} \delta^{\beta \gamma}+\right.$ permut. $)$ & $\frac{5}{16} \partial_{r}^{7}+5 \alpha_{3} \partial_{r}^{2}$ \\
\hline \hline
\end{tabular}

TABLE I: Examples for the symmetric traceless tensors $\mathcal{S}_{l}$ Eq. (18) and for the differential operators $\mathcal{D}_{l}$ in Eq. 13. $n^{\alpha}$ are the components of the unit vector $\vec{n}=\vec{r} / r$.

we adopt the standard assumption of implicit summation over repeated indexes, the three directions of space $\{x, y, z\}$ are labeled by a Greek index $\alpha \in\{1,2,3\}$ and $[\alpha \beta \ldots]$ denotes a set of $l$ indexes. The idea is to use the symmetric tensors $\mathcal{S}_{l}$ which appear in the multipolar expansion used in electrostatics [44]. They can be defined through their components by:

$$
\mathcal{S}_{l[\alpha \beta \ldots]}=(-1)^{l} \frac{r^{l+1}}{(2 l-1) ! !} \partial_{[\alpha \beta \ldots]}^{l} \frac{1}{r},
$$

where we have introduced a short-hand notation for the l-th partial derivatives:

$$
\partial_{[\alpha \beta \ldots]}^{l}=\frac{\partial}{\partial x^{\alpha}} \frac{\partial}{\partial x^{\beta}} \ldots,
$$

and $\left\{x^{\alpha}\right\}$ are the three components of the radial vector $\vec{r}$ in the Cartesian basis $\left\{\vec{e}_{\alpha}\right\}$ : i.e. $\vec{r}=x^{\alpha} \vec{e}_{\alpha}$. These tensors can be expressed in terms of the component $n^{\alpha}$ of the unit vector $\vec{n}=\vec{r} / r$ and are functions of the angles $\Omega$ only. The normalization factor in Eq.(18) has been chosen for later convenience and explicit expressions of $\mathcal{S}_{l}$ and $\mathcal{D}_{l}$ with $l \leq 3$ are given in Tab.(II). In the following, we will use some useful properties of these tensors which are collected in the Appendix. By construction, $\mathcal{S}_{l}$ are traceless $\left(\delta_{\alpha \beta} \mathcal{S}_{l}^{[\alpha \beta \ldots]}=0\right)$ and symmetric tensors of rank $l$. They are eigenvectors of $\hat{L}^{2}(\hat{L}$ is the angular momentum operator) with eigenvalue $\hbar^{2} l(l+1)$. Similarly to the spherical harmonics $Y^{l m}(\Omega)$, the tensors $\mathcal{S}_{l}$ can serve as a basis for an alternative multipolar expansion of a wave function, like in Eq.(15). To this end, we define the Cartesian representation of the multipolar radial functions of order $l$ associated with a wave function $\Psi$, by a symmetric trace free tensor $\mathcal{C}_{\Psi, l}(r)$ having the components:

$$
\mathcal{C}_{\Psi, l}^{[\alpha \beta \ldots]}(r)=\frac{r^{l+1}}{l !} \int_{\Omega} \frac{d^{2} \Omega}{4 \pi}\left(\mathcal{S}_{l}^{[\alpha \beta \ldots]} \Psi(r, \Omega)\right)
$$

For example, the monopolar $(l=0)$ function $\mathcal{C}_{\Psi, 0}(r)$ is just $r$ times the projection of the wave function in the $s$-wave channel. Quite generally, $\mathcal{C}_{\Psi, l}^{[\alpha \beta \ldots]}(r)$ are regular functions of $r$ analogous to the coefficients $c_{\Psi}^{l m}(r)$ in Eq. (15) and characterize the behavior of the wave function $\Psi(r, \Omega)$ in the channel $l$ through the expansion (see 
Eqs.(6972) in the Appendix):

$$
\left\langle\vec{r}\left|\Pi_{l}\right| \Psi\right\rangle=\frac{(2 l+1) ! !}{r^{l+1}} \mathcal{S}_{l[\alpha \beta \ldots]} \mathcal{C}_{\Psi, l}^{[\alpha \beta \ldots]}(r) .
$$

Using these notations, the generalized Bethe-Peierls boundary conditions Eq. (16) can be written as:

$$
\lim _{r \rightarrow 0}\left(\mathcal{D}_{l}+\frac{1}{w_{l}}\right) \mathcal{C}_{\Psi, l}^{[\alpha \beta \ldots]}(r)=0 .
$$

Eqs. 2012122) are equivalent to Eqs. (1516). We show in the next section that they permit to find a general form of the pseudopotential.

Note that an alternative formulation of the condition (22) in the $s$-wave channel amounts to imposing the following singularity on the two-body wave function:

$$
\left\langle\vec{r}\left|\Pi_{0}\right| \psi\right\rangle=\frac{\mathcal{C}_{\Psi, 0}(0)}{r}\left(1-\frac{r}{a}+A_{0}\left(r^{2}+2 \alpha_{0} r\right)\right)+\mathcal{O}\left(r^{3}\right) .
$$

In the $p$-wave channel $l=1$, the short-range behavior of the interacting two-body wave function takes also a simple form:

$$
\left\langle\vec{r}\left|\Pi_{1}\right| \Psi\right\rangle=\frac{\vec{p} \cdot \vec{r}}{r^{3}}\left(1-\frac{r^{3}}{3 \mathcal{V}_{s}}+A_{1}\left(3 r^{2}-2 \alpha_{1} r^{3}\right)\right)+\mathcal{O}\left(r^{2}\right),
$$

where the "dipolar momentum" $\vec{p}$ defined by $p^{\alpha}=$ $3 \mathcal{C}_{\Psi, 1}^{\alpha}(0)$, characterizes the $p$-wave singularity of the wave function. In general situations the coefficients $\left(A_{0}, A_{1}, \mathcal{C}_{\Psi, 0}(0), \vec{p}\right)$ can be functions of time and of the center of mass coordinates of the two particles.

\section{Zero-range pseudopotential}

Instead of giving a final expression of the pseudopotential directly, we prefer to detail the different steps in its derivation. Such a way of presentation can be useful if one wants to derive pseudopotentials associated with others expressions of the phase shift in Eq.(44) or in different contexts (low dimensions for example). In either cases, a zero-range pseudopotential can be deduced from the three following constraints: $(i)$ it cancels the delta terms arising from action of the Laplacian on the singularities of the wave function at $r=0$ in the Schrödinger equation, (ii) it imposes the correct boundary conditions and (iii) it can be used in the Born approximation. The first condition (item- $i$ ) can be fulfilled by introducing potential terms in the different channels $l$ of the form (see Eq.(77) in the Appendix):

$$
\begin{aligned}
\left\langle\vec{r}\left|V_{l}^{(i)}\right| \Psi\right\rangle=(-1)^{l+1} & \frac{2 \pi(2 l+1) \hbar^{2}}{\mu} \\
& \mathcal{C}_{\Psi, l}^{[\alpha \beta \ldots]}(0)\left(\partial_{[\alpha \beta \ldots]}^{l} \delta\right)(\vec{r}) .
\end{aligned}
$$

In order to include the boundary conditions Eqs.(22) (item-ii), we consider the "boundary operator" $\mathcal{B}_{l}$ defined by:

$$
\left\langle\vec{r}\left|\mathcal{B}_{l}\right| \Psi\right\rangle=\left(\partial_{[\alpha \beta \ldots]}^{l} \delta\right)(\vec{r}) \lim _{r \rightarrow 0}\left(\mathcal{D}_{l}+\frac{1}{w_{l}}\right) \mathcal{C}_{\Psi, l}^{[\alpha \beta \ldots]}(r) .
$$

By construction wave functions belonging to the kernel of $\mathcal{B}_{l}$ satisfy the desired boundary conditions. Consequently, the family of pseudopotentials defined by:

$$
V_{l}^{(i)}+\beta \mathcal{B}_{l}
$$

where $\beta$ is an arbitrary parameter, can be used to solve the two-body problem in the resonant regime. Interestingly, while exact zero-range solutions can be obtained $\forall \beta \neq 0$, it is not hard to be convinced that a first order Born approximation performed for example in the computation of the two-body scattering states leads to a $\beta$-dependent result. Indeed in this approximation, the two-body wave function $\Psi^{(0)}$ is an eigenstate of the noninteracting Schrödinger equation (a plane wave in the example chosen) and is then a regular wave function of the radius $r$. Consequently, the action of $V_{l}^{(i)}$ on $\Psi^{(0)}$ gives a zero result while $\left\langle\vec{r}\left|V_{l}^{(i)}+\beta \mathcal{B}_{l}\right| \Psi^{(0)}\right\rangle$ is $\beta$-dependent. Furthermore, and we will show this property explicitly in the next section, varying $\beta$ offers the freedom to adjust the pseudopotential in such a way that the first order Born approximation is exact at a given collisional energy (item-iii). Finally, the expression of the pseudopotential in Eq. (27) although general, can be rewritten in the more convenient following form:

$$
\left\langle\vec{r}\left|V_{\lambda, l}\right| \Psi\right\rangle=(-1)^{l} g_{\lambda, l}\left(\partial_{[\alpha \beta \ldots]}^{l} \delta\right)(\vec{r}) \mathcal{R}_{\lambda, l}^{[\alpha \beta \ldots]}[\Psi] .
$$

In this expression, $g_{\lambda, l}=(-1)^{l} \beta$ is the coupling constant defined by:

$$
g_{\lambda, l}=\frac{2 \pi(2 l+1) \hbar^{2} w_{l}}{\mu\left(1-\lambda w_{l}\right)}
$$

and $\mathcal{R}_{\lambda, l}[$.$] are symmetric trace free tensors which gener-$ alize the regularizing operator introduced in Refs. [8, 35]. They act on a wave function $\Psi$ as:

$$
\mathcal{R}_{\lambda, l}^{[\alpha \beta \ldots]}[\Psi]=\lim _{r \rightarrow 0}\left(\mathcal{D}_{l}+\lambda\right) \mathcal{C}_{\Psi, l}^{[\alpha \beta \ldots]}(r)
$$

The parameter $\lambda$ appears in Eq.(29) and Eq.(30) both, and by construction (like $\beta$ in Eq.(27)) it is a free parameter of the theory: exact wave functions obtained in the zero-range formalism do not depend on the value of $\lambda$. The pseudopotentials in Eq.(28) generalize for arbitrary partial wave channels the $s$-wave and $p$-wave $\lambda$-potentials already introduced in Ref. [8, 35].

\section{SOME ILLUSTRATIONS AND PROPERTIES OF THE PSEUDOPOTENTIAL}

\section{A. Two-body scattering states from the Green's method}

This section is both an illustration of the formalism and a way to test the proposed form of the pseudopotential given in Eq.(28). We consider the case where two 
particles interact in the $l$ th partial wave channel without external potential and we derive the scattering states from the general Green's function method. The interacting two-body wave function $\Psi_{\vec{k}}(\vec{r}$ ) (wave vector $\vec{k}$, energy $\left.E=\hbar^{2} k^{2} / 2 \mu\right)$ is then the solution of the integral equation:

$$
\Psi_{\vec{k}}(\vec{r})=\Psi_{\vec{k}}^{(0)}(\vec{r})-\int d^{3} \vec{r}^{\prime} G_{E}\left(\vec{r}, \vec{r}^{\prime}\right)\left\langle\vec{r}^{\prime}\left|V_{\lambda, l}\right| \Psi_{\vec{k}}\right\rangle
$$

where $\Psi_{\vec{k}}^{(0)}(\vec{r})=\exp (i \vec{k} \cdot \vec{r})$ is the incident plane wave and $G_{E}$ is the one-body outgoing free Green's function at energy $E$ :

$$
G_{E}\left(\vec{r}, \vec{r}^{\prime}\right)=\frac{\mu}{2 \pi \hbar^{2}} \frac{\exp i k u}{u} \quad \text { with } \quad u=\left|\vec{r}-\vec{r}^{\prime}\right| \text {. }
$$

Integrating Eq.31) over $\vec{r}^{\prime}$ coordinates gives:

$$
\begin{aligned}
\Psi_{\vec{k}}(\vec{r})=\Psi_{\vec{k}}^{(0)}(\vec{r})-\frac{\mu g_{\lambda, l}}{2 \pi \hbar^{2}} \mathcal{R}_{\lambda, l}^{[\alpha \beta \ldots]}\left[\Psi_{\vec{k}}\right] \\
\left(\partial_{[\alpha \beta \ldots]}^{\prime l} \frac{\exp i k u}{u}\right)_{r^{\prime}=0},
\end{aligned}
$$

where $\partial_{[\alpha \beta \ldots]}^{\prime l}$ stands for the $l$ th partial derivatives with respect to $\vec{r}^{\prime}$ coordinates. Thanks to the trace free property $\left(\delta_{\alpha \beta} \mathcal{R}_{\lambda, l}^{[\alpha \beta \ldots]}\left[\Psi_{\vec{k}}\right]=0\right)$, contribution of the $l$ th derivative in Eq. (33) takes a simple form. Indeed, for a given function $F(u)$, one can show that:

$$
\begin{aligned}
& \left(\partial_{[\alpha \beta \ldots]}^{\prime l} F(u)\right)_{r^{\prime}=0} \mathcal{R}_{\lambda, l}^{[\alpha \beta \ldots]}\left[\Psi_{\vec{k}}\right]= \\
& \left(n_{\alpha} n_{\beta} \ldots\right) \mathcal{R}_{\lambda, l}^{[\alpha \beta \ldots]}\left[\Psi_{\vec{k}}\right](-r)^{l}\left(\frac{1}{r} \partial_{r}\right)^{l} F(r),
\end{aligned}
$$

and with $F(u)=G_{E}\left(\vec{r}, \vec{r}^{\prime}\right)$, Eq.

$$
\begin{gathered}
\Psi_{\vec{k}}(\vec{r})=\Psi_{\vec{k}}^{(0)}(\vec{r})-\frac{\mu g_{\lambda, l}}{2 \pi \hbar^{2}} \mathcal{S}_{l[\alpha \beta \ldots]} \mathcal{R}_{\lambda, l}^{[\alpha \beta \ldots]}\left[\Psi_{\vec{k}}\right] \\
(-r)^{l}\left(\frac{1}{r} \partial_{r}\right)^{l}\left(\frac{\exp (i k r)}{r}\right) .
\end{gathered}
$$

As $r \rightarrow 0$, one recognizes a multipolar singularity in the two-body wave function:

$$
\Psi_{\vec{k}}(\vec{r})=-\frac{(2 l+1) ! ! w_{l}}{\left(1-\lambda w_{l}\right)} \frac{\mathcal{S}_{l[\alpha \beta \ldots]}}{r^{l+1}} \mathcal{R}_{\lambda, l}^{[\alpha \beta \ldots]}\left[\Psi_{\vec{k}}\right]+\mathcal{O}\left(r^{1-l}\right),
$$

and comparison with Eq.(21) gives the multipole components:

$$
\mathcal{C}_{\Psi_{\vec{k}}, l}^{[\alpha \beta \ldots]}(0)=-\frac{w_{l}}{1-\lambda w_{l}} \mathcal{R}_{\lambda, l}^{[\alpha \beta \ldots]}\left[\Psi_{\vec{k}}\right]
$$

In the partial wave channels of angular quantum number different from $l$, components of the wave function $\Psi_{\vec{k}}(\vec{r})$ coincide as expected with the partial waves of $\Psi_{\vec{k}}^{(0)}(\vec{r})$. All the information on the interaction are gathered in the term $\mathcal{S}_{l[\alpha \beta \ldots]} \mathcal{R}_{\lambda, l}^{[\alpha \beta \ldots]}\left[\Psi_{\vec{k}}\right]$ in Eq.(35). From Eq.(21), one can see that it contains implicitly the projector $\Pi_{l}$ through:

$$
\begin{aligned}
& \mathcal{S}_{l[\alpha \beta \ldots]} \mathcal{R}_{\lambda, l}^{[\alpha \beta \ldots]}[\Psi]= \\
& \frac{1}{(2 l+1) ! !} \lim _{r \rightarrow 0}\left(\mathcal{D}_{l}+\lambda\right) r^{l+1}\left\langle\vec{r}\left|\Pi_{l}\right| \Psi\right\rangle .
\end{aligned}
$$

A closed equation is obtained by applying the operator $\mathcal{S}_{l[\alpha \beta \ldots]} \mathcal{R}_{\lambda, l}^{[\alpha \beta \ldots][.]}$ on both sides of Eq. (35). Using the usual decomposition of a plane wave over spherical ones:

$$
\exp (i \vec{k} \cdot \vec{r})=\sum_{l=0}^{\infty} i^{l}(2 l+1) \mathcal{P}_{l}\left(\vec{n} \cdot \vec{n}_{k}\right) j_{l}(k r)
$$

together with Eq. (9) this leads to:

$$
\begin{aligned}
\lim _{r \rightarrow 0}\left(\mathcal{D}_{l}+\lambda\right) r^{l+1}\left\langle\vec{r}\left|\Pi_{l}\right| \Psi_{\vec{k}}^{(0)}\right\rangle= \\
(2 l+1) ! !(i k)^{l} \mathcal{P}_{l}\left(\vec{n} . \vec{n}_{k}\right),
\end{aligned}
$$

on the other hand:

$$
\begin{aligned}
\lim _{r \rightarrow 0}\left(\mathcal{D}_{l}+\lambda\right) r^{2 l+1}\left(\frac{1}{r} \partial_{r}\right)^{l}\left(\frac{\exp (i k r)}{r}\right)= \\
(-1)^{l}(2 l-1) ! !\left(\lambda+\alpha_{l} k^{2}+i k^{2 l+1}\right),
\end{aligned}
$$

and finally, one gets:

$$
\mathcal{S}_{l[\alpha \beta \ldots]} \mathcal{R}_{\lambda, l}^{[\alpha \beta \ldots]}\left[\Psi_{\vec{k}}\right]=\frac{\left(1-\lambda w_{l}\right)(i k)^{l} \mathcal{P}_{l}\left(\vec{n} \cdot \vec{n}_{k}\right)}{1+w_{l} \alpha_{l} k^{2}+i w_{l} k^{2 l+1}} .
$$

As expected, the expression of the scattering wave function does not depend on the parameter $\lambda$ :

$$
\begin{array}{r}
\Psi_{\vec{k}}(\vec{r})=\Psi_{\vec{k}}^{(0)}(\vec{r})-\frac{(2 l+1) w_{l} \mathcal{P}_{l}\left(\vec{n} \cdot \vec{n}_{k}\right)}{1+w_{l} \alpha_{l} k^{2}+i w_{l} k^{2 l+1}} \\
(-i k r)^{l}\left(\frac{1}{r} \partial_{r}\right)^{l}\left(\frac{\exp (i k r)}{r}\right) .
\end{array}
$$

Asymptotically for $k r \gg 1$, the two-body wave function is given by:

$$
\Psi_{\vec{k}}(\vec{r})=\exp (i \vec{k} \cdot \vec{r})+(2 l+1) \mathcal{P}_{l}\left(\vec{n} . \vec{n}_{k}\right) f_{l} \frac{\exp (i k r)}{r},
$$

where the partial amplitude $f_{l}$ is given by Eq. (5).

It is interesting to note that the exact result can be also obtained at the level of the first order Born approximation (which is a " $\lambda$-dependent" treatment) by choosing a particular value of the parameter $\lambda$ :

$$
\lambda^{\text {Born }}=-\alpha_{l} k^{2}-i k^{2 l+1} .
$$

For example by setting $\lambda=0$ in a first order Born approximation gives a correct evaluation of a scattering process at a vanishly small collisional energy (Wigner threshold).

Generalization to the case where the interaction occurs in several partial wave channels is straightforward: it is obtained after simple addition of the corresponding pseudopotentials given by Eq.(28). 


\section{B. Links with other formalisms}

It is instructive to compute the matrix element of $V_{\lambda, l}$ between two states $|\Phi\rangle$ and $|\Psi\rangle$. A simplifying step is to realize that the projection operator in the partial wave $(l)$ is implicitly present in the expression of the pseudopotential Eq.(28). Indeed, assuming that $\Phi(\vec{r})$ is regular at $r=0$, one obtains:

$$
\mathcal{S}_{l}^{[\alpha \beta \ldots]}\left(\partial_{[\alpha \beta \ldots]}^{l} \Phi\right)_{r=0}=l ! \lim _{r \rightarrow 0} \frac{\left\langle\vec{r}\left|\Pi_{l}\right| \Phi\right\rangle}{r^{l}} .
$$

Moreover from Eqs. (3846) and Eq.(73) in the Appendix, one finds:

$$
\begin{aligned}
&\left\langle\Phi\left|V_{\lambda, l}\right| \Psi\right\rangle=g_{\lambda, l} \int \frac{d^{2} \Omega}{4 \pi}\left(\lim _{r \rightarrow 0} \frac{\left\langle\Phi\left|\Pi_{l}\right| \vec{r}\right\rangle}{r^{l}}\right. \\
&\left.\lim _{r \rightarrow 0}\left(\mathcal{D}_{l}+\lambda\right) r^{l+1}\left\langle\vec{r}\left|\Pi_{l}\right| \Psi\right\rangle\right) .
\end{aligned}
$$

First, we consider plane waves $\langle\vec{r} \mid \vec{k}\rangle=\exp (i \vec{k} . \vec{r})$. The matrix element between two such states is:

$$
\left\langle\vec{k}^{\prime}\left|V_{\lambda, l}\right| \vec{k}\right\rangle=g_{\lambda, l}\left(k k^{\prime}\right)^{l} \mathcal{P}_{l}\left(\vec{n}_{k}{ }^{\prime} \cdot \vec{n}_{k}\right),
$$

with $\vec{n}_{k}{ }^{\prime}=\vec{k}^{\prime} / k^{\prime}$. For wave vectors of same modulus $\left(k=k^{\prime}=\sqrt{2 \mu E} / \hbar\right)$ and for the particular value of the free parameter $\lambda$ given in Eq. [45], comparison with the expression of the partial wave amplitude Eq.(5) shows that Eq. (48) coincides with the on-shell $T$-matrix $t\left(\vec{k}^{\prime} \leftarrow\right.$ $\vec{k})$ taken at energy $E$ :

$$
t\left(\vec{k}^{\prime} \leftarrow \vec{k}\right)=\left\langle\vec{k}^{\prime}\left|V_{\lambda \text { Born }, l}\right| \vec{k}\right\rangle=-\frac{2 \pi \hbar^{2}}{\mu} f\left(\vec{k}^{\prime} \leftarrow \vec{k}\right),
$$

where $f\left(\vec{k}^{\prime} \leftarrow \vec{k}\right)=(2 l+1) \mathcal{P}_{l}\left(\vec{n}_{k}{ }^{\prime} \cdot \vec{n}_{k}\right) f_{l}(k)$ is the full scattering amplitude.

Another interesting application of Eq.(47) is given by considering spherical outgoing waves:

$$
\langle\vec{r} \mid k l m\rangle=Y^{l m}(\Omega) j_{l}(k r) \quad .
$$

In this case, the matrix elements are given by:

$$
\left\langle k l m\left|V_{\lambda, l}\right| k^{\prime} l^{\prime} m^{\prime}\right\rangle=\delta_{l l^{\prime}} \delta_{m m^{\prime}} \frac{g_{\lambda, l}\left(k k^{\prime}\right)^{l}}{4 \pi(2 l+1)} .
$$

For $\lambda=0$, this result coincides with the one obtained in Ref. [45]. Here, the $\lambda$-freedom is a new ingredient and shows clearly that mean field calculations in zero-range approaches have to be developed carefully. For example, considering a two-component Fermi gas close to the unitary limit with $\alpha_{0}=0$ and a large and negative value of the scattering length $w_{0}$, the mean field model in Ref. 45] predicts an instability which is not observed in experiments 28, 29, 30, 31, 32. This failure can be explained as follows. The interaction concerns only particles close to the Fermi surface (Pauli blocking) with a Fermi wave vector $k_{F}$. However, in this mean field approach the approximation made on the interaction term is only valid for binary processes of vanishly small colliding energies (equivalent to a Born approximation with the pseudopotential and the particular choice $\lambda=0$ ). Consequently in the resonant regime where $k_{F}\left|w_{0}\right| \gtrsim 1$, the characteristic collisional energy of pairs of particles on the Fermi surface is $\hbar^{2} / m w_{0}^{2}$ and the approximation made is not justified. By the way, it is possible to build a qualitative and simple mean field model which supports the stability of the gas at the BCS-BEC crossover [46].

Taking two states with angular parts characterized by the same spherical harmonic in Eq.477), one obtains the expression of the pseudopotential in radial coordinates:

$$
v_{\lambda, l}=\frac{g_{\lambda, l}}{4 \pi} \frac{\delta(r)}{r^{l+2}} \lim _{r \rightarrow 0}\left(\mathcal{D}_{l}+\lambda\right)\left(r^{l+1} .\right)
$$

In the particular case $\lambda=0, \alpha_{l}=0$, r.h.s. of Eq.(52) coincides with the form of the pseudopotential introduced in Ref. [37]:

$$
v_{0, l}=\frac{\hbar^{2} w_{l}(2 l+1) ! !}{2 \mu(2 l) ! !} \frac{\delta(r)}{r^{l+2}} \lim _{r \rightarrow 0} \partial_{r}^{2 l+1}\left(r^{l+1} .\right) .
$$

Note that the delta-shell regularization procedure introduced in Ref. 37] while correct appears not to be essential in the zero-range approach.

\section{Regularized scalar product}

Except for the case of the Fermi pseudopotential which is a particular case of Eq.(28), two scattering states $\Psi_{\vec{k}}$, and $\Psi_{\vec{k}}$ (with $\vec{k} \neq \vec{k}^{\prime}$ ) of expressions given by Eq. (43) are not orthogonal each others with respect to the usual scalar product. To examine this point, we exclude the singularity at $r=0$ in the scalar product between the two different scattering states by introducing a cut-off $r_{0}$. We obtain from the Schrödinger equation:

$$
\begin{aligned}
& \int_{r>r_{0}} d^{3} \vec{r} \Psi_{\vec{k}}^{*}(\vec{r}) \Psi_{\vec{k}}(\vec{r})=\frac{r_{0}^{2}}{k^{2}-k^{\prime 2}} \\
& \quad \int_{r=r_{0}} d^{2} \Omega \Psi_{\vec{k}}^{*}(\vec{r}) \partial_{r} \Psi_{\vec{k}}(\vec{r})-\Psi_{\vec{k}}(\vec{r}) \partial_{r} \Psi_{\vec{k}^{\prime}}^{*}(\vec{r})
\end{aligned}
$$

This relation is general i.e. it is also valid for scattering wave functions associated with a finite range two-body potential whatever the value of $r_{0}$. In standard situations where the Hamiltonian is hermitian, the r.h.s. of (54) is zero for $r_{0}=0$. However in the zero-range approach, for wave functions satisfying the boundary conditions in Eq. (13), as $r_{0} \rightarrow 0$ this term converges toward a constant value for $l=0$ and $\alpha_{0} \neq 0$ and even worse, it diverges for $l>0$. Interestingly, the regular part of Eq.(54) for such wave functions can be obtained through the following 
surface integration:

$$
\begin{aligned}
& \operatorname{Reg}_{r_{0} \rightarrow 0}\left\{\int_{r>r_{0}} d^{3} \vec{r} \Psi_{\vec{k}}^{*}(\vec{r}) \Psi_{\vec{k}}(\vec{r})\right\}= \\
& \quad-\lim _{r_{0} \rightarrow 0} \sum_{l=0}^{\infty} \frac{\alpha_{l} r_{0}^{2 l+2}}{((2 l-1) ! !)^{2}} \int_{r=r_{0}} d^{2} \Omega \Psi_{\vec{k}}^{*}(\vec{r})\left\langle\vec{r}\left|\Pi_{l}\right| \Psi_{\vec{k}}\right\rangle
\end{aligned}
$$

where the operator $\operatorname{Reg}_{r_{0} \rightarrow 0}\{\cdot\}$ extracts the regular part at $r_{0}=0$. The meaning of this result is that the Laplacian operator is not Hermitian with respect to the usual scalar product when one considers singular functions like the ones in Eq. (43). In another hand, the true scattering states associated with the finite range potential experienced by the particles, are orthogonal to each other so that direct identification of the true scattering states with the states $\left\{\left|\Psi_{\vec{k}}\right\rangle\right\}$ is not possible. This feature follows from the fact that the mapping between the two eigen basis is not valid for $r \lesssim R$ while it is justified only outside the potential range. Indeed, the singular boundary behavior imposed on wave functions in Eq.(16) is a way to reproduce the effect of the true finite range potential for $r>R$ but has a formal character for interparticle distances $r \lesssim R$. In order to understand this issue more closely, we consider the sphere of radius $R$ which delimits the outer parts (region $r>R$ ) from the inner parts (region $r<R$ ) of the true wave functions. Contribution to the scalar product coming from the outer-part of the true wave functions is given by Eq. (54) with $r_{0}=R$ and due to orthogonality between the true scattering states, scalar product of the inner-parts cancels this term. However in the zero-range potential approach the inner parts of the wave functions $(r<R)$ are not properly described, so that compensation of the two contributions does not occur. To solve this inconsistency, the idea is to modify the usual scalar product itself by including implicitly the contribution of the inner parts. We define then a regularized scalar product $(. \mid .)_{0}$ by subtracting the surface term (r.h.s. of Eq.(54) ) from the usual scalar product as $r_{0} \rightarrow 0:$

$$
\begin{aligned}
& (\Psi \mid \Phi)_{0}=\operatorname{Reg}_{r_{0} \rightarrow 0}\left\{\int_{r>r_{0}} d^{3} \vec{r} \Psi^{*}(\vec{r}) \Phi(\vec{r})\right\} \\
& \quad+\lim _{r_{0} \rightarrow 0} \sum_{l=0}^{\infty} \frac{\alpha_{l} r_{0}^{2 l+2}}{((2 l-1) ! !)^{2}} \int_{r=r_{0}} d^{2} \Omega \Psi^{*}(\vec{r})\left\langle\vec{r}\left|\Pi_{l}\right| \Phi\right\rangle
\end{aligned}
$$

so that by construction, two eigenstates of the zero-range Hamiltonian are orthogonal to each other with respect to this metrics.

In general there are several possible representations of Eq.(56) due to the various and equivalent ways of extracting a regular part. We give below explicit expressions of the regularized scalar product associated with the pseudopotential (28) in the $l=0$ and $l=1$ partial wave channels:
- Particles interacting in the $s$-wave channel only:

$$
\begin{aligned}
(\Psi \mid \Phi)_{0}=\lim _{r_{0} \rightarrow 0} & \left\{\int_{r>r_{0}} d^{3} \vec{r} \Psi^{*}(\vec{r}) \Phi(\vec{r})\right. \\
& \left.+\alpha_{0} r_{0}^{2} \int_{r=r_{0}} d^{2} \Omega \Psi^{*}(\vec{r}) \Phi(\vec{r})\right\} .
\end{aligned}
$$

This result can be written more formally by introducing a scalar product of the form:

$$
(\Psi \mid \Phi)_{0}=\int d^{3} \vec{r} g(r) \Psi^{*}(\vec{r}) \Phi(\vec{r})
$$

with the weight $g(r)=1+\alpha_{0} \delta(r)$.

- Particles interacting in the $p$-wave channel only (for example in the case of polarized fermions):

$$
\begin{aligned}
(\Psi \mid \Phi)_{0}= & \lim _{r_{0} \rightarrow 0}\left\{\int_{r>r_{0}} d^{3} \vec{r} \Psi^{*}(\vec{r}) \Phi(\vec{r})\right. \\
& \left.+\left(\alpha_{1} r_{0}^{4}-r_{0}^{3}\right) \int_{r=r_{0}} d^{2} \Omega \Psi^{*}(\vec{r}) \Phi(\vec{r})\right\} .
\end{aligned}
$$

This scalar product has been first introduced in Ref. 35] in the same form as in Eq. (58) with the weight $g(r)=1+\delta(r)\left[\left(\alpha_{1} r^{2}-r\right)\right.$. $]$.

\section{Bound states and their normalizations}

In this section, we consider the normalization of the two-body bound states of vanishing energy supported by the zero-range pseudopotential. We show that using the regularized scalar product is equivalent to the method based on the analyticy of the scattering amplitude [38]. This last method was used in Ref. 15] in the context of $s$-wave narrow resonances. In this case or also for $p$ wave or higher partial waves channel resonance where bound states are even not square integrable with respect to the usual scalar product (they don't belong to the $\mathcal{L}^{2}$ Hilbert space), the regularized scalar product appears as the natural way to perform the normalization in the configuration space. Moreover, this scalar product allows for a generalization of the method of Ref. [38] in the case of inhomogeneous situations.

If one considers solutions of the Schrödinger equation with a finite range potential as functions of $\vec{r}$ and of the energy $E$ : $\Psi(E, \vec{r})$, then a bound state of energy $E_{B}$ and wave function $\Psi_{B}=\Psi\left(E_{B}, \vec{r}\right)$ corresponds to a case where $\Psi(E, \vec{r})$ is square integrable. Using the Schrödinger equation, one can show that:

$$
\begin{aligned}
\int_{r<r_{0}} d^{3} \vec{r}\left|\Psi_{B}\right|^{2}= & -\frac{\hbar^{2} r_{0}^{2}}{2 \mu} \int_{r=r_{0}} d^{2} \Omega\left(\Psi^{*}(E, \vec{r}) \partial_{r} \partial_{E} \Psi(E, \vec{r})\right. \\
& \left.-\partial_{r} \Psi^{*}(E, \vec{r}) \partial_{E} \Psi(E, \vec{r})\right)_{E=E_{B}} .
\end{aligned}
$$

Using this identity permits to deduce that the normalization of a bound state is linked to the residue of the 
scattering amplitude at $E=E_{B}$ [38]. Using Eq.(60), the norm of the state $\Psi(E, \vec{r})$ can be also written as:

$$
\begin{array}{r}
\langle\Psi \mid \Psi\rangle=\int_{r>R} d^{3} \vec{r}|\Psi|^{2}-\frac{\hbar^{2} R^{2}}{2 \mu} \int_{r=R} d^{2} \Omega\left(\Psi^{*} \partial_{r} \partial_{E} \Psi\right. \\
\left.-\partial_{r} \Psi^{*} \partial_{E} \Psi\right) .
\end{array}
$$

The link between Eq. (61) and the result given by the regularized scalar product appears by noticing that Eq. (60) is exactly the opposite of the r.h.s. of Eq. (54) in the limit $k^{\prime} \rightarrow k$ and for $r_{0}=R$. As a result, the norm of a state in the zero-range scheme obtained through Eq.(56) coincides with the formal limit $R \rightarrow 0$ in Eq. 61).

In the absence of an external potential, wave functions of two body bound states of energy $E_{B}=-\hbar^{2} \kappa^{2} / 2 \mu$ in channel $(l)$ are of the form (for $r>R$ ):

$$
\Psi_{B}(r, \Omega)=\mathcal{N}_{l} \phi_{l}(\Omega) r^{l}\left(\frac{1}{r} \partial_{r}\right)^{l}\left(\frac{\exp -\kappa r}{r}\right),
$$

where $\phi_{l}(\Omega)$ is a normalized angular function. The expression for the bound state energy is found by solving $1 / f_{l}(i \kappa)=0$. For a partial scattering amplitude given by Eq.(5), one finds in the resonant regime i.e. for large and positive values of $w_{l}$, a bound state of vanishing energy with $\kappa^{2} \simeq 1 / w_{l} \alpha_{l}$ (obviously in the case of the $s$-wave broad resonance $\alpha_{0}=0$ and $\left.\kappa=1 / w_{0}\right)$. This is the state which plays a crucial role in the BCS-BEC crossover. In the zero-range approach, Eq. 62] is formally extended in the domain $0 \leq r \leq R$ and using the regularized scalar product, one finds that for $\left(\Psi_{B} \mid \Psi_{B}\right)_{0}=1$ :

$$
\mathcal{N}_{l}^{2}=\frac{1}{\alpha_{l}+(-1)^{l}\left(l+\frac{1}{2}\right) \kappa^{(2 l-1)}} .
$$

This result is very interesting as it coincides with the one given by the residue of $f_{l}$ at the energy $E=E_{B}$ [38].

To conclude this section, we show that the scalar product (56) gives a constraint on the possible values of the "effective range" parameter $\alpha_{l}$ compatible with a consistent description of the low-energy bound state and hence of the resonant regime. Indeed, the probability of finding the molecular state outside the potential range is less than one: $\int_{r>R} d^{3} \vec{r}\left|\Psi_{B}\right|^{2}<1$. Then, using Eq. [63) and keeping the dominant contribution as $R \rightarrow 0$, one finds that the parameter $\alpha_{l}$ verifies in the resonant regime for $l>0$ :

$$
\alpha_{l} R^{2 l-1} \gtrsim(2 l-3) ! !(2 l-1) ! ! \quad .
$$

In the case of a narrow $s$-wave resonance, in the regime of intermediate detuning $\left(\alpha_{0} \gg w_{0}\right)$ and $w_{0} \gtrsim R$, then $\kappa^{2} \simeq 1 / w_{0} \alpha_{0}$ and the condition $\alpha_{0} \gg R$ follows directly from $\kappa R \ll 1$. By the way, Eq. 64 implies also that $\alpha_{l}>0$. As an example, the experimental fit performed in 29] for a particular $p$-wave resonance is compatible with Eq. (64). Let us note that the ideal case for a description of the bound state within the zero-range approach corresponds to the situation where the two terms in Eq. 64 are almost equal. In this regime the probability of finding the dimer in the region $r>R$, i.e. in the region described by the zero-range model, is maximum.

\section{CONCLUSIONS}

In this paper, we have developed a general zero-range one channel model for resonant scattering in arbitrary partial waves. In this approach, the wave function is a solution of the free Schrödinger equation and the interaction is replaced by energy independent boundary conditions on the wave function as the distance between the two interacting particles goes formally to zero. This way, we generalize the Bethe-Peierls approach which has been widely used in the case of broad $s$-wave resonances. We have shown, how the boundary conditions can be implemented in the Schrödinger equation through zero-range pseudopotentials. In a given partial wave channel, the interaction can be described either in terms of a boundary condition on the wave function or with a family of pseudopotentials generated by an extra degree of freedom (the parameter $\lambda$ ). By construction, the Schrödinger equation is invariant under a change of the parameter $\lambda$. This transformation appears then as a general symmetry of zero-range potential approaches. The pseudopotentials derived in this paper are the generalization of $\lambda$-potential already obtained in the $s$-wave and $p$-wave channels 8, 35]. This class of pseudopotentials can serve as source terms in the Schrödinger equation permits us to search solutions with the Green's functions method. While exact treatments are invariant under a change of the $\lambda$ parameter, this property is in general no longer true in approximate schemes. This gives in turn a way to improve the approximations made (this idea can be applied in mean field treatments and has been already used in the Hartree-Fock-Bogoliubov formalism for the Bose gas [8, 9]). For example, we have shown that with a specific choice of $\lambda$, the first order Born approximation gives the exact result for two-body scattering states at a finite colliding energy.

Using the fact that the scattering states are not mutually orthogonal in the zero-range scheme (except for the Fermi pseudopotential), we have shown how the notion of a regularized scalar product emerges naturally. Normalization of bound states by means of this metrics gives in free space the same result as the method based on the analyticy of the scattering amplitude. As it applies directly in configuration space, this new tool gives us a simple way to perform the normalization in inhomogeneous situations.

The formalism presented in this paper can be used to obtain the scattering amplitude of particles confined in linear or planar atomic waveguides, thus allowing for studies of ultracold gases trapped in low dimensions. The few-body problem is another direction of research where this approach should be fruitful.

Note added: Recently we learned of recent related works by Idziaszek and Calarco [47] and also Derevianko [48] where the form of the pseudopotential in Eq. (53) is also found. The main difference of the present approach with respect to Ref. 47] (or also Ref. [37]) is that the pseudopotential in Eq.(28) or equivalently the general- 
ized Bethe-Peierls conditions in Eq. (16) have been derived in an energy independent form.

\section{ACKNOWLEDGMENTS}

Y. Castin, F. Chevy, M. Holzmann and F. Werner are acknowledged for thorough discussions on the subject. Laboratoire de Physique Théorique de la Matière Condensée is Unité Mixte de Recherche 7600 of Centre National de la Recherche Scientifique.

\section{APPENDIX: USEFUL PROPERTIES OF THE SYMMETRIC TRACE FREE TENSORS $\mathcal{S}_{l}$}

This appendix collects some useful properties of the symmetric trace free tensors $\mathcal{S}_{l}$ defined in Eq.(18) 44, 49]. Let us consider the two vectors $\vec{r}=x^{\alpha} \vec{e}_{\alpha}$ and $\vec{r}^{\prime}=$ $x^{\prime \alpha} \vec{e}_{\alpha}$. By definition:

$$
\frac{1}{\left|\vec{r}-\vec{r}^{\prime}\right|}=\sum_{l=0}^{\infty} \frac{(2 l-1) ! !}{l !} \mathcal{S}_{l[\alpha \beta \ldots]} \frac{x^{\prime \alpha} x^{\prime \beta} \ldots}{r^{l+1}} .
$$

Introducing the unit vectors $\vec{n}=\vec{r} / r=n^{\alpha} \vec{e}_{\alpha}, \vec{n}^{\prime}=$ $\vec{r}^{\prime} / r^{\prime}=n^{\prime \alpha} \vec{e}_{\alpha}$ and the symmetric trace free tensor $\mathcal{S}_{l}^{\prime}$ associated with $\vec{r}^{\prime}$, we have:

$$
\mathcal{S}_{l[\alpha \beta \ldots]} n^{\prime \alpha} n^{\prime \beta} \ldots=\mathcal{S}_{l[\alpha \beta \ldots]}^{\prime} \mathcal{S}_{l}^{[\alpha \beta \ldots]},
$$

so that Eq. (65) can be rewritten

$$
\frac{1}{\left|\vec{r}-\vec{r}^{\prime}\right|}=\frac{1}{r} \sum_{l=0}^{\infty} \mathcal{S}_{l[\alpha \beta \ldots]} \mathcal{S}_{l}^{\prime[\alpha \beta \ldots]} \frac{(2 l-1) ! !}{l !}\left(\frac{r^{\prime}}{r}\right)^{l} .
$$

This expression can be identified with the standard expansion (for $r^{\prime}<r$ ) [50]:

$$
\frac{1}{\left|\vec{r}-\vec{r}^{\prime}\right|}=\frac{1}{r} \sum_{l=0}^{\infty}\left(\frac{r^{\prime}}{r}\right)^{l} \mathcal{P}_{l}\left(\vec{n} \cdot \vec{n}^{\prime}\right)
$$

where $\mathcal{P}_{l}(x)$ is the Legendre polynomial of degree $l$ and we obtain:

$$
\mathcal{P}_{l}\left(\vec{n} . \vec{n}^{\prime}\right)=\frac{(2 l-1) ! !}{l !} \mathcal{S}_{l[\alpha \beta \ldots]} \mathcal{S}_{l}^{\prime[\alpha \beta \ldots]} .
$$

For example, taking $\vec{n}^{\prime}=\vec{e}_{z}$ shows that:

$$
\mathcal{S}_{l(z z z \ldots z)}=\frac{l !}{(2 l-1) ! !} \mathcal{P}_{l}(\cos \theta)
$$

with $\theta=\left(\widehat{\vec{r}, \vec{e}_{z}}\right)$. Eq. (69) can be used to have an expression of the projection operator $\Pi_{l}$ over the partial wave $l$ in terms of the tensor $\mathcal{S}_{l}$. For that purpose, we recall the addition theorem [50]:

$$
\mathcal{P}_{l}\left(\vec{n} \cdot \vec{n}^{\prime}\right)=\frac{4 \pi}{2 l+1} \sum_{m=-l}^{m=l} Y^{l m}(\Omega) Y^{l m *}\left(\Omega^{\prime}\right)
$$

where $\left(\Omega=(\theta, \phi), \Omega^{\prime}=\left(\theta^{\prime}, \phi^{\prime}\right)\right)$ are the angles defining the spherical coordinates for the two vectors $\left(\vec{r}, \vec{r}^{\prime}\right)$. The projection of a wave function $\Psi$ reads:

$$
\left\langle\vec{r}\left|\Pi_{l}\right| \Psi\right\rangle=(2 l+1) \int \frac{d^{2} \Omega^{\prime}}{4 \pi} \Psi\left(r, \Omega^{\prime}\right) \mathcal{P}_{l}\left(\vec{n} . \vec{n}^{\prime}\right)
$$

together with Eq. (69) and the definition of the multipoles of the wave function Eq.(20), we obtain Eq.(21).

In this paper, we also use an interesting property of the symmetric traceless tensors found in Ref. [49]. Let us consider two symmetric and traceless tensors $\mathcal{A}$ and $\mathcal{B}$ of order $l$, then

$$
\begin{array}{r}
\mathcal{A}^{[\alpha \beta \ldots]}\left(\int \frac{d^{2} \Omega^{\prime}}{4 \pi} n_{\alpha}^{\prime} n_{\beta}^{\prime} \ldots n_{\alpha^{\prime}}^{\prime} n_{\beta^{\prime}}^{\prime}\right) \mathcal{B}^{\left[\alpha^{\prime} \beta^{\prime} \ldots\right]} \\
=\frac{l !}{(2 l+1) ! !} \mathcal{A}_{[\alpha \beta \ldots]} \mathcal{B}^{[\alpha \beta \ldots]} .
\end{array}
$$

As an example, taking $\mathcal{A}=\mathcal{S}_{l}$ and $\mathcal{B}=\mathcal{S}_{l}^{\prime \prime}$ allows one to recover from Eqs. 6973 ) the known relation:

$$
\mathcal{P}_{l}\left(\vec{n} \cdot \vec{n}^{\prime \prime}\right)=(2 l+1) \int \frac{d^{2} \Omega^{\prime}}{4 \pi} \mathcal{P}_{l}\left(\vec{n} \cdot \vec{n}^{\prime}\right) \mathcal{P}_{l}\left(\vec{n}^{\prime} . \vec{n}^{\prime \prime}\right)
$$

We close this appendix with the link between the singularity of the $l$ th partial wave of a wave function with the derivatives of the delta distribution. To this end, we use the so-called relation:

$$
\Delta_{\vec{r}}\left(\frac{1}{\left|\vec{r}-\vec{r}^{\prime}\right|}\right)=-4 \pi \delta\left(\vec{r}-\vec{r}^{\prime}\right)
$$

together with Eq.(67) and the formal expansion:

$$
\delta\left(\vec{r}-\vec{r}^{\prime}\right)=\sum_{l=0}^{\infty} \frac{\left(-r^{\prime}\right)^{l}}{l !}\left(\partial_{[\alpha \beta \ldots]}^{l} \delta\right)(\vec{r}) n^{\prime \alpha} n^{\prime \beta} \ldots
$$

where $\left(\partial_{[\alpha \beta \ldots]}^{l} \delta\right)(\vec{r})$ is the $l$ th partial derivative of the delta distribution (see Eq.(19)). Finally, we obtain:

$$
\begin{aligned}
& \Delta_{\vec{r}}\left(\frac{\mathcal{S}_{l[\alpha \beta \ldots]}}{r^{l+1}}\right) n^{\prime \alpha} n^{\prime \beta} \ldots= \\
& 4 \pi \frac{(-1)^{l+1}}{(2 l-1) ! !}\left(\partial_{[\alpha \beta \ldots]}^{l} \delta\right)(\vec{r}) n^{\prime \alpha} n^{\prime \beta} \ldots
\end{aligned}
$$

This expression justifies the introduction of the potential $V_{l}^{(i)}$ in Eq.(25). Using Eq.(69), one can also write Eq.(77) in terms of Legendre Polynomials:

$$
\Delta_{\vec{r}}\left(\frac{\mathcal{P}_{l}(\cos \theta)}{r^{l+1}}\right)=4 \pi \frac{(-1)^{l+1}}{l !}\left(\partial_{z}^{l} \delta\right)(\vec{r})
$$


[1] H. Bethe and R. Peierls, Proc. R. Soc. London, Ser. A 148, 146 (1935).

[2] E. Fermi, Ric. Sci. 7-II, 13 (1936).

[3] G. Breit, Phys. Rev. 71, 215 (1947).

[4] J.M. Blatt and V.F. Weisskopf, in Theoretical Nuclear Physics, Wiley, New York (1952).

[5] C. Cohen-Tannoudji, Course at Collège de France, Lectures 4,5 (1998-99). http://www.phys.ens.fr/cours/college-de-france/1998-99/19

[6] Y. Castin, in Coherent Atomic Matter Waves, Lecture Notes of Les Houches Summer School, EDP Sciences and (Springer-Verlag, Berlin, 2001), pp.1-136.

[7] G. Bruun, Y. Castin, R. Dum and K. Burnett, Eur. Phys. J. D 7, 433 (1999).

[8] M. Olshanii and L. Pricoupenko, Phys. Rev. Lett. 88, 010402 (2002).

[9] L. Pricoupenko, Phys. Rev. A 70, 013601 (2004).

[10] D.S. Petrov, D.M. Gangardt and G.V. Shlyapnikov, J. Phys. IV France 64, 5 (2004), and references therein.

[11] M.G. Moore, T. Bergeman and M. Olshanii, J. Phys. IV France 116, 69 (2004), and references therein.

[12] V. Peano, M. Thorwart, C. Mora, and R. Egger, New J. Phys. 7, 192 (2005).

[13] D.S. Petrov, Phys. Rev. A 67, 010703(R) (2003).

[14] D.S. Petrov, C. Salomon, and G.V. Shlyapnikov, Phys. Rev. A 71, 012708(R) (2005).

[15] D.S. Petrov, Phys. Rev. Lett. 93, 143201 (2004).

[16] C. Mora, R. Egger, A.O. Gogolin, and A. Komnik, Phys. Rev. Lett. 93, 170403 (2004).

[17] S. Inouye, M.R. Andrews, J. Stenger, H.-J. Miesner, D.M. Stamper-Kurn, and W. Ketterle, Nature (London) 392, 151 (1998).

[18] S. Jochim, M. Bartenstein, A. Altmeyer, G. Hendl, S. Riedl, C. Chin, J. Hecker Denschlag, and R. Grimm, Science 302, 2101 (2003).

[19] M.W. Zwierlein, C.A. Stan, C.H. Schunck, S.M.F. Raupach, S. Gupta, Z. Hadzibabic, and W. Ketterle, Phys. Rev. Lett. 91, 250401 (2003).

[20] T. Bourdel, L. Khaykovich, J. Cubizolles, J. Zhang, F. Chevy, M. Teichmann, L. Tarruell, S.J.J.M.F. Kokkelmans, and C. Salomon, Phys. Rev. Lett. 93, 050401 (2004).

[21] J. Kinast, S.L. Hemmer, M.E. Gehm, A. Turlapov, and J.E. Thomas, Phys. Rev. Lett. 92, 150402 (2004).

[22] Markus Greiner, Cindy A. Regal, and Deborah S. Jin, Nature (London) 426, 537 (2003).

[23] Y. Castin, C. R. Phys. 5, 407 (2004).

[24] F. Werner and Y. Castin, cond-mat/0507399.

[25] M.W. Zwierlein, J.R. Abo-Shaeer, A. Schirotzek, C.H. Schunck, and W. Ketterle, Nature (London) 435, 1047 (2005).

[26] P. Nozières and S. Schmitt-Rink, J. Low Temp. Phys. 59, 195 (1985).

[27] M. Randeria, in Bose-Einstein Condensation, edited by
A. Griffin, D. W. Snoke, S. Stringari (Cambridge University Press, Cambridge, England, 1995), p.355.

[28] C.A. Regal, C. Ticknor, J.L. Bohn, and D.S. Jin, Phys. Rev. Lett. 90, 053201 (2003).

[29] C. Ticknor, C.A. Regal, D.S. Jin, and J.L. Bohn, Phys. Rev. A 69, 042712 (2004).

[30] J. Zhang, E.G.M. van Kempen, T. Bourdel, L. Khaykovich, J. Cubizolles, F. Chevy, M. Teichmann, 98-99.htmlarruell, S.J.J.M.F. Kokkelmans, and C. Salomon, Phys. Rev. A 70, 030702(R) (2004).

[31] C.H. Schunck, M.W. Zwierlein, C.A. Stan, S.M.F. Raupach, W. Ketterle, A. Simoni, E. Tiesinga, C.J. Williams, and P.S. Julienne, Phys. Rev. A 71, 045601 (2005).

[32] F. Chevy, E.G.M. Van Kempen, T. Bourdel, J. Zhang, L. Khaykovich, M. Teichmann, L. Tarruell, S.J.J.M.F. Kokkelmans, and C. Salomon, Phys. Rev. A 71, 062710 (2005).

[33] T. Volz, S. Dür, N. Syassen, G. Rempe, E.G.M. van Kempen, and S.J.J.M.F. Kokkelmans, Phys. Rev. A 72, 010704(R) (2005).

[34] J. Stenger, S. Inouye, M.R. Andrews, H.-J. Miesner, D.M. Stamper-Kurn, and W. Ketterle, Phys. Rev. Lett. 82, 2422 (1999).

[35] L. Pricoupenko, cond-mat/0505448

[36] K. Huang and C. N. Yang, Phys. Rev. 105, 767 (1956).

[37] R. Stock, A. Silberfarb, E.L. Bolda and I.H. Deutsch, Phys. Rev. Lett. 94, 023202 (2005).

[38] L. Landau et E. Lifchitz, Tome III Mécanique Quantique, Editions MIR Moscou (1967).

[39] J. Dalibard, in Proceedings of the International School of Physics "Enrico Fermi", edited by M. Inguscio, S. Stringari and C. Wieman, Course CXL (IOS, Amsterdam, 1999), pp.321-349.

[40] M. Marinescu, H.R. Sadeghpour and A. Dalgarno, Phys. Rev. A 49, 982 (1994).

[41] N.F. Mott and M.S. Massey, in The Theory of Atomic Collisions, 3rd ed. (Clarendon, Oxford, 1965).

[42] H. Feshbach, Ann. Phys. (N.Y.) 19, 287 (1962).

[43] M. Abramowitz and Irene Stegun, in Handbook of Mathematical Functions (Dover Publications, Inc., New York, 1974).

[44] R. Courant and D. Hilbert, in Methods of Mathematical Physics Vol. I (John Wiley, New-York,1989).

[45] R. Roth and H. Feldmeier, Phys. Rev. A 64, 043603 (2001).

[46] L. Pricoupenko and Y. Castin, Phys. Rev. A 69, 051601(R) (2004).

[47] Z. Idziaszek and T. Calarco, quant-ph/0507186

[48] A. Derevianko, Phys. Rev. A 72, 044701 (2005).

[49] K.S. Thorne, Rev. Mod. Phys. 52, No 2, Part I, 299 (1980).

[50] P.M. Morse and H. Feshbach, in Methods of Theoretical Physics (Mc Graw-Hill, New-York, 1953). 